\title{
Urinary bisphenol $A$ and obesity in adults: results from the Canadian Health Measures Survey
}

\author{
Minh T. Do, PhD (1,2,3); Vicky C. Chang, MPH (1,2); Michelle A. Mendez, PhD (4); Margaret de Groh, PhD (1)
}

This article has been peer reviewed.

\begin{abstract}
Introduction: Exposure to bisphenol A (BPA) has been shown to affect lipid metabolism and promote weight gain in animal studies. Recent epidemiological studies also support a link between BPA and obesity in human populations, although many were limited to a single adiposity measure or have not considered potential confounding by dietary factors. The purpose of this study is to examine associations between urinary BPA and adiposity measures in a nationally representative sample of Canadian adults.
\end{abstract}

Methods: We performed analyses using biomonitoring and directly measured anthropometric data from 4733 adults aged 18 to 79 years in the Canadian Health Measures Survey (2007-2011). We used multinomial and binary logistic regression models to estimate associations of urinary BPA with body mass index (BMI) categories (overweight vs. under/normal weight; obesity vs. under/normal weight) and elevated waist circumference (males: $\geq 102 \mathrm{~cm}$; females: $\geq 88 \mathrm{~cm}$ ), respectively, while controlling for potential confounders. Linear regression analyses were also performed to assess associations between urinary BPA and continuous BMI and waist circumference measures.

Results: Urinary BPA was positively associated with BMI-defined obesity, with an odds ratio of 1.54 (95\% confidence interval [CI]: 1.002-2.37) in the highest (vs. lowest) BPA quartile (test for trend, $p=.041$ ). Urinary BPA was not associated with elevated waist circumference defined using standard cut-offs. Additionally, each natural-log unit increase in urinary BPA concentration was associated with a $0.33 \mathrm{~kg} / \mathrm{m}^{2}$ (95\% CI: 0.100.57 ) increase in BMI and a $1.00 \mathrm{~cm}$ (95\% CI: 0.34-1.65) increase in waist circumference.

Conclusion: Our study contributes to the growing body of evidence that BPA is positively associated with obesity. Prospective studies with repeated measures are needed to address temporality and improve exposure classification.

Keywords: bisphenol A, endocrine disruptors, obesity, body mass index, waist circumference, biomonitoring, Canadian Health Measures Survey

\section{Introduction}

Obesity is a growing epidemic worldwide and is expected to exceed smoking as a leading contributor to the burden of chronic disease. ${ }^{1,2}$ In Canada, one in four adults is obese. ${ }^{3}$ Excess consumption of energy-dense foods, inadequate physical activity, and increased sedentary behaviour have been identified as the most important factors contributing to the obesity epidemic. ${ }^{4}$

Given the dramatic increase in obesity over the past several decades, additional hypotheses are being explored to identify other potentially modifiable risk factors beyond the energy imbalance equation. Ecological studies initially reported a correlation between increasing obesity

\section{Highlights}

- This is the first Canadian study to investigate the association between bisphenol A (BPA) and adiposity measures

- A higher level of urinary BPA is associated with greater odds of being obese among Canadian adults 18 to 79 years of age.

- Urinary BPA concentration is also positively associated with continuous measures of adiposity, including body mass index and waist circumference.

- Prospective studies with repeated measures are needed to address temporality and improve exposure classification.

prevalence and increasing production of synthetic chemicals, including bisphenol A (BPA). ${ }^{5}$ BPA is an endocrine-disrupting chemical commonly found in food and beverages stored in polycarbonate plastic and epoxy resin containers. ${ }^{6}$ The primary route of exposure to BPA is through dietary intake, although dermal exposure can also occur from skin contact with thermal paper (e.g. receipts, tickets). ${ }^{6}$ More than $90 \%$ of Canadians ${ }^{7}$ and Americans ${ }^{8}$ have detectable levels of BPA in their urine, indicating widespread exposure in human populations.

In vitro studies have shown that BPA enhances adipocyte cell differentiation, leading to excess fat accumulation. ${ }^{9}$ Rodent studies have also found BPA exposure to increase adipose tissue mass and promote weight gain. $^{10}$ More recently, 
evidence of a relationship between BPA and obesity in humans has emerged but is limited to a small number of populations. To date, epidemiological studies in the USA, $^{11,12}$ China, ${ }^{13}$ and Korea ${ }^{14,15}$ have reported positive associations between BPA and adiposity measures in adults. Moreover, as an estrogen-like compound, BPA has been shown to affect males and females differently in animal models, ${ }^{16}$ although evidence of sex differences in the association between BPA and obesity in humans is limited.

Since 2007, the Canadian Health Measures Survey (CHMS) has been collecting biomonitoring data and anthropometric measures from a nationally representative sample of Canadians. Given widespread exposure to BPA in Canada and existing evidence supporting the link between BPA and obesity, we evaluated associations between urinary BPA and measures of adiposity in adults aged 18 to 79 years using CHMS data, overall and by sex.

\section{Methods}

\section{Data source and study population}

This study utilized data from cycles 1 (2007-2009) and 2 (2009-2011) of the CHMS. Details of the CHMS have been described elsewhere. ${ }^{17,18}$ Briefly, the CHMS is an ongoing cross-sectional survey that uses a multistage stratified sampling design to collect nationally representative data of the Canadian household population, excluding persons living on reserves or other Aboriginal settlements, full-time members of the Canadian Forces, and residents of institutions and certain remote regions. Cycle 1 collected data from 5604 respondents aged 6 to 79 years at 15 sites across Canada; cycle 2 collected data from 6395 respondents aged 3 to 79 years at 18 sites. The sample for each cycle represented approximately $96 \%$ of the Canadian population in the target age range.

The CHMS consists of an in-home household interview capturing sociodemographic, lifestyle, and health characteristics, followed by a mobile examination centre (MEC) visit for direct physical measures, including anthropometric measurements and collection of blood and urine samples. Written informed consent was obtained from each respondent. Protocols were reviewed and approved by Health Canada and the Public Health Agency of Canada (PHAC)'s Research Ethics Board.
Analyses for this study were restricted to adults aged 18 to 79 years at the time of the MEC visit (cycle 1: $\mathrm{n}=3726$; cycle 2: $\mathrm{n}=3873$ ). We pooled data from two CHMS cycles in order to increase sample size and statistical precision of estimates. ${ }^{19}$ While all respondents providing urine samples in cycle $1(n=3702)$ were eligible for measurements of BPA, only a random subset of cycle 2 respondents $(n=1117)$ was selected for these measures, resulting in a combined sample of 4819 respondents. We further excluded pregnant women ( $\mathrm{n}=37$ ) and respondents with missing urinary BPA and/or creatinine ( $\mathrm{n}=49$ ), yielding a final sample of 4733 respondents.

\section{Laboratory measurements}

Single spot urine samples were collected as midstream urine in cycle 1 and firstcatch urine in cycle 2 . Cycle 2 respondents were also asked to refrain from urinating 2 hours prior to the MEC visit. The protocol was modified to accommodate new tests for infectious disease markers introduced in cycle $2 .{ }^{18}$ Despite these changes, urinary BPA levels were similar for the two cycles. ${ }^{6}$ After collection and aliquoting, urine samples were frozen at $-20^{\circ} \mathrm{C}$ and shipped on dry ice to the testing laboratory at the Institut national de santé publique du Québec (Quebec, Canada) for analysis.

Concentrations of total BPA (free and conjugated) were measured using gas chromatography-tandem mass spectrometry based on previously described methods.,20 The limit of detection (LOD) was $0.2 \mu \mathrm{g} / \mathrm{L}$ for both cycles. Concentrations below the LOD ( $n=436$; $9 \%$ ) were assigned a value of LOD/2 $(0.1 \mu \mathrm{g} / \mathrm{L})$. We categorized urinary BPA concentrations into quartiles ( $<0.7 \mu \mathrm{g} / \mathrm{L}, 0.7$ to $1.2 \mu \mathrm{g} / \mathrm{L}, 1.3$ to $2.4 \mu \mathrm{g} / \mathrm{L}$, and $>2.4 \mu \mathrm{g} / \mathrm{L}$ ) based on distribution in the overall study population. Additionally, BPA concentrations were natural-log transformed due to skewed distribution and analyzed as a continuous variable.

Urinary creatinine was used to adjust for urine dilution and was measured using the colorimetric end-point Jaffe method. ${ }^{21}$ Concentrations below the LOD (cycle 1: $0.035 \mathrm{~g} / \mathrm{L}$; cycle $2: 0.050 \mathrm{~g} / \mathrm{L}$ ) were coded as missing and excluded from the analyses $(n=9)$ as per Statistics Canada's guidelines. ${ }^{19}$ To reduce potential bias associated with systematic differences in urinary creatinine concentrations across population characteristics (e.g. sex, age, race/ethnicity), we included creatinine as a covariate in all models instead of standardizing BPA concentrations for creatinine. $^{22}$

\section{Anthropometric measurements}

Our primary outcome of interest was body mass index (BMI), derived from height and weight measured using standard procedures. ${ }^{23}$ Standing height was measured to the nearest $0.01 \mathrm{~cm}$ using a ProScale M150 digital stadiometer (Accurate Technology Inc., Fletcher, NC, USA). Weight was measured to the nearest 0.1 kg using a Mettler Toledo VLC with Panther Plus terminal scale (Mettler Toledo Canada, Mississauga, ON, Canada). BMI, which was calculated as weight (kg) divided by height squared $\left(\mathrm{m}^{2}\right)$, was classified into the following categories: underweight $\left(<18.5 \mathrm{~kg} / \mathrm{m}^{2}\right)$, normal weight (18.5 to $24.9 \mathrm{~kg} / \mathrm{m}^{2}$ ), overweight (25.0 to $\left.29.9 \mathrm{~kg} / \mathrm{m}^{2}\right)$, obesity class I $(30.0$ to $34.9 \mathrm{~kg} / \mathrm{m}^{2}$ ), and obesity class II or III $\left(\geq 35.0 \mathrm{~kg} / \mathrm{m}^{2}\right) .^{4}$

As an indicator of abdominal fat, we also examined waist circumference, which was measured to the nearest $0.1 \mathrm{~cm}$ at the end of a normal expiration at the mid-point between the bottom of the rib cage and the top of the iliac crest. ${ }^{4}$ Central obesity was defined using sex-specific waist circumference cut-offs: $\geq 102 \mathrm{~cm}$ in males and $\geq 88 \mathrm{~cm}$ in females. ${ }^{4,24}$

\section{Potential confounders}

We identified potential confounders according to previous literature examining the BPA-obesity association in adults. ${ }^{11-15}$ Sociodemographic variables included sex; age (18 to 29,30 to 39,40 to 49,50 to 59 , 60 to 69 , or $\geq 70$ years); race/ethnicity (white or non-white); highest level of education (less than secondary school degree, secondary school degree, or post-secondary degree); and household income adequacy (low/lower middle, upper middle, or high), categorized based on annual household income and number of people living in the household. We also considered lifestyle factors, including smoking status (never, former, or current); alcohol consumption ( 0 to 3 times/month, 1 to 6 times/week, or daily); and physical activity, which was assessed based on average daily energy expenditure during leisuretime activities reported over the past 3 months and categorized as active 
( $\geq 3.0 \mathrm{kcal} / \mathrm{kg} /$ day), moderately active (1.5 to $2.9 \mathrm{kcal} / \mathrm{kg} / \mathrm{day})$, or inactive ( $<1.5 \mathrm{kcal} / \mathrm{kg} /$ day $)$.

Given that dietary intake is the primary source of BPA exposure and also a known risk factor for obesity, several dietary measures to control for potential confounding were developed utilizing the semi-quantitative food frequency questionnaire in the CHMS, which asked the respondents to report the number of times (per day, week, month, or year) certain types of foods or drinks were consumed over the past year. A diet quality score was derived using a similar approach as the construction of a Mediterranean diet index. ${ }^{25}$ First, we grouped food items into seven components (fruits; vegetables; legumes and nuts; cereals and grains; milk/dairy products; fish and seafood; and red/processed meat) and calculated sex-specific median intakes (times per day) for each. With the exception of red/processed meat, all components were considered beneficial and assigned a value of ' 1 ' when intake levels were above the median (red/processed meat intake below the median was assigned ' 1 '). The overall diet quality score was determined by summing up values across all components and was categorized as low ( 0 to 3 ), medium (4 to 5 ), or high (6 to 7). In addition, we also examined consumption frequencies of foods or drinks that potentially contain BPA and/or are known to be "obesogenic" due to their high-energy content. These included sugar-sweetened beverages (e.g. regular/non-diet soft drinks, sport drinks, fruit-flavoured drinks), categorized as $<1$ time/week, 1 to 6 times/week, or $\geq 1$ time/day; and junk food (e.g. French fries, regular-fat potato/tortilla/corn chips, ice cream), categorized as $<1$ time/week, 1 to 4 times/week, or $\geq 5$ times/week.

\section{Statistical analysis}

To account for the complex sampling design of the CHMS, sampling weights were used in all our analyses. ${ }^{17,18}$ Variance estimates were obtained using the bootstrap method. Analyses were performed using SAS version 9.3 (SAS Institute Inc., Cary, NC, USA) and SUDAAN version 11.0.1 (Research Triangle Institute, Research Triangle Park, NC, USA). Statistical significance was evaluated at $p<.05$, and tests were two-sided.
Descriptive analyses were conducted to examine distributions of characteristics in the total population and by BPA quartile. The Rao-Scott modified chi-square test was used to determine whether the distribution of BPA quartiles differed across categories of each characteristic. Geometric mean (GM) urinary BPA concentrations were also calculated, and differences were assessed using the $t$-test with Bonferroniadjusted $p$-values depending on the number of comparisons.

We used multinomial logistic regression to examine associations between urinary BPA quartiles and BMI, categorized as under/normal weight $\left(<25.0 \mathrm{~kg} / \mathrm{m}^{2}\right.$; reference category), overweight (25.0 to $\left.29.9 \mathrm{~kg} / \mathrm{m}^{2}\right)$, or obesity $\left(\geq 30.0 \mathrm{~kg} / \mathrm{m}^{2}\right)$. Tests for trend across quartiles were performed by modelling the median of each BPA quartile as a continuous variable. We also examined natural log-transformed BPA concentration as a continuous variable in a separate model. Furthermore, to assess the association between urinary BPA and central obesity (i.e. elevated waist circumference), binary logistic regression was performed. All basic models adjusted for sex, age (continuous years),

TABLE 1

Geometric mean and distribution of urinary bisphenol A concentrations across population characteristics among Canadian adults 18 to 79 years of age $(n=4733)$, 2007-2011 Canadian Health Measures Survey

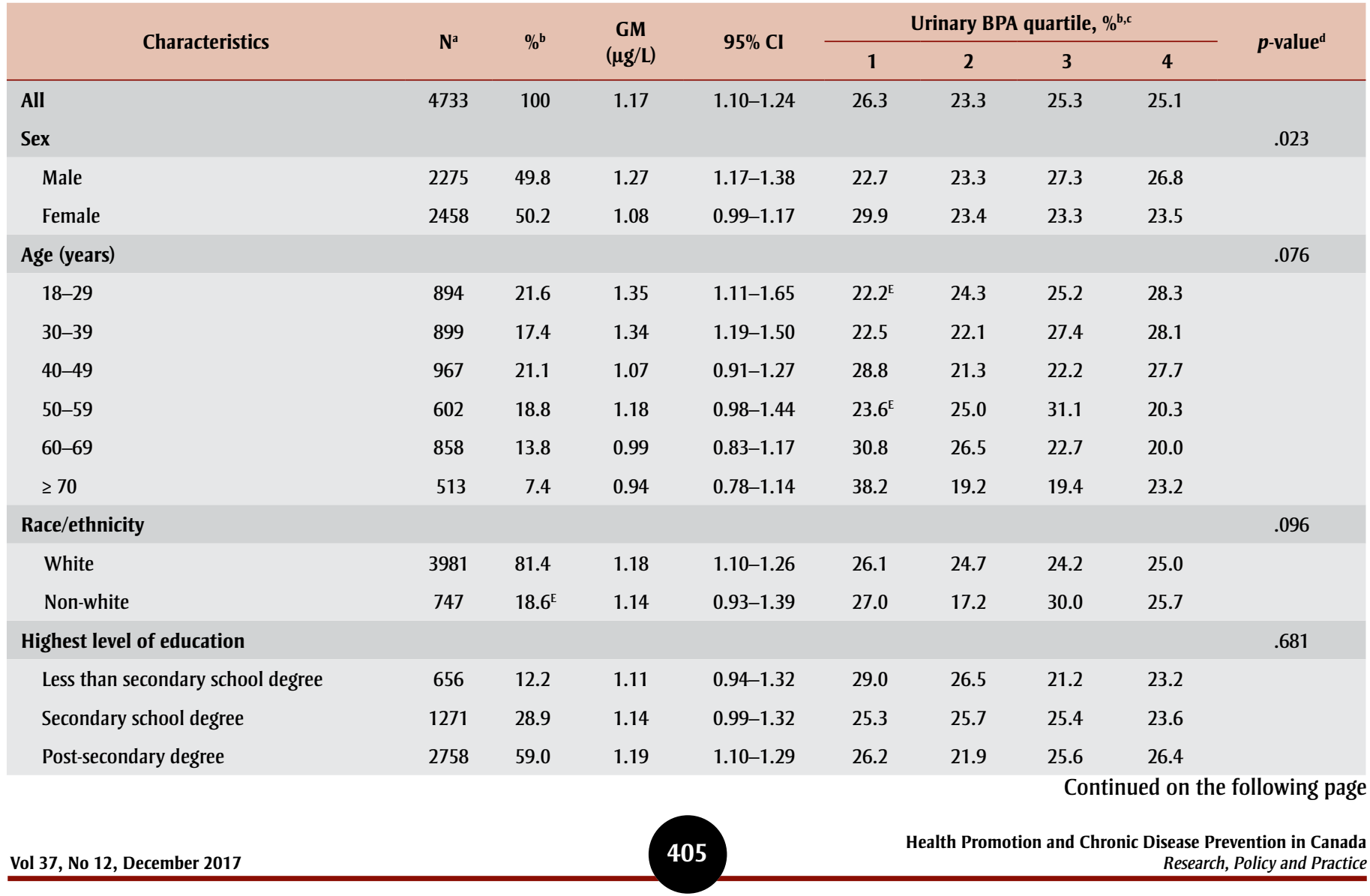


TABLE 1 (continued)

Geometric mean and distribution of urinary bisphenol A concentrations across population characteristics among Canadian adults 18 to 79 years of age $(n=4733)$, 2007-2011 Canadian Health Measures Survey

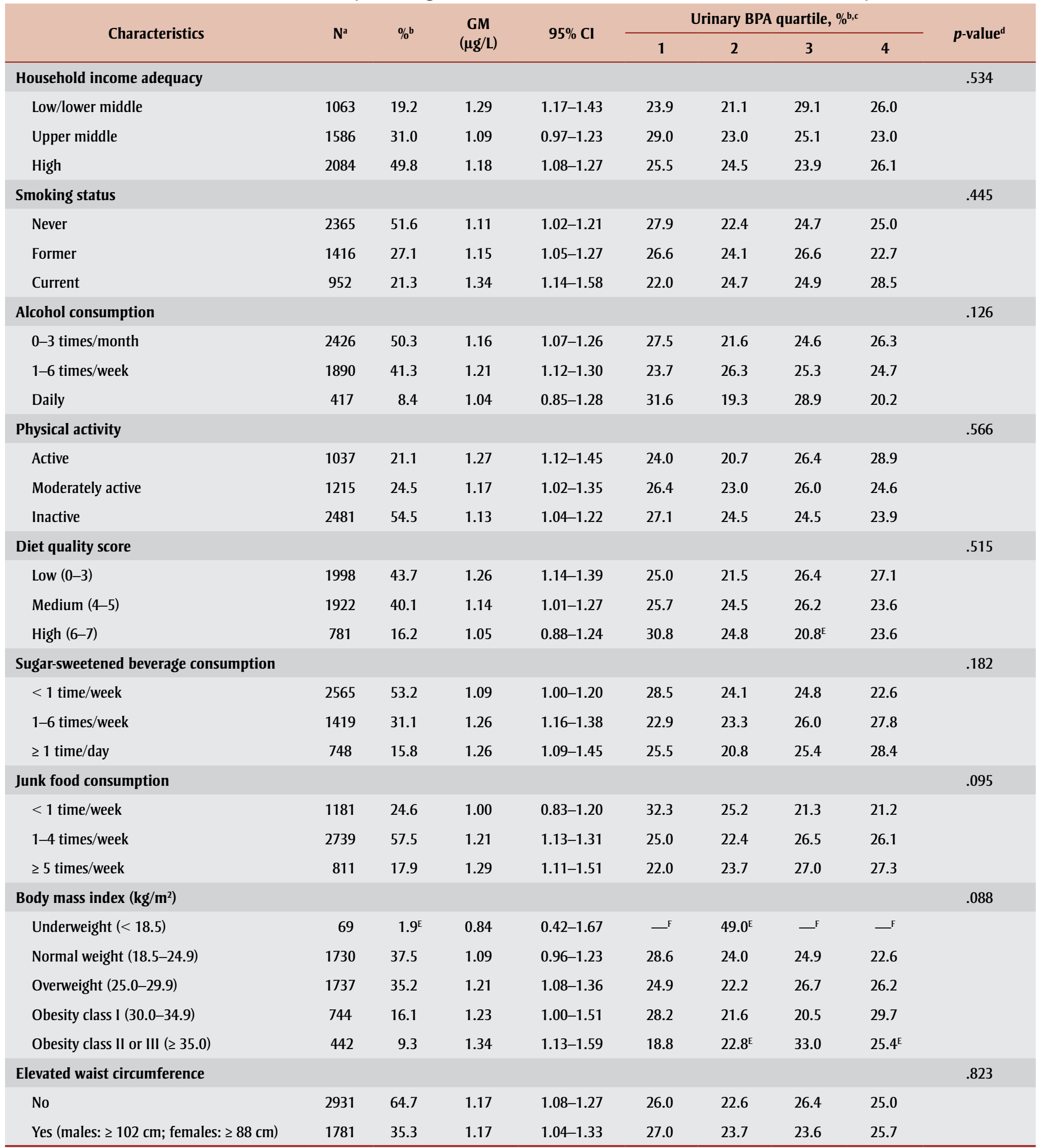

Abbreviations: BPA, bisphenol A; Cl, confidence interval; GM, geometric mean.

Note: Percentages and GMs were weighted using sampling weights.

${ }^{\text {a }}$ Numbers may not sum up to the total $(n=4733)$ due to missing data for some variables.

${ }^{\text {b }}$ Percentages may not sum up to $100 \%$ due to rounding.

${ }^{\text {c }}$ Quartile 1: $<0.7 \mu \mathrm{g} / \mathrm{L}$; quartile 2: 0.7-1.2 $\mu \mathrm{g} / \mathrm{L}$; quartile 3: 1.3-2.4 $\mu \mathrm{g} / \mathrm{L}$; quartile 4: $>2.4 \mu \mathrm{g} / \mathrm{L}$.

${ }^{\mathrm{d}} p$-value from the Rao-Scott modified chi-square test comparing the distribution of BPA quartiles across categories of each characteristic.

${ }^{\mathrm{E}}$ Interpret with caution (coefficient of variation is between $16.6 \%$ and $33.3 \%$ ).

F Data do not meet Statistics Canada's guidelines for release due to extreme variability (coefficient of variation > 33.3\%). 
and urinary creatinine concentration (continuous g/L). We then constructed multivariate models by adding individual variables from the list of potential confounders to the basic models. Variables were included in the final models if they were associated with both the exposure (BPA quartile; $p<.20$ from chi-square test) and the outcome (BMI or waist circumference category; entered the regression model at $p<.20$ ), or if they changed the sex-, age-, and creatinine-adjusted odds ratio (OR) for BPA by $>10 \%$. In addition to sex, age, and urinary creatinine, all BMI and waist circumference models adjusted for race/ethnicity, alcohol consumption, and junk food consumption; waist circumference models additionally adjusted for sugar-sweetened beverage consumption. We also estimated associations of urinary BPA (as quartiles or natural log-transformed continuous variable) with continuous measures of BMI and waist circumference using linear regression. Additionally, we stratified our analyses to explore potential effect modification by sex. Statistical significance of multiplicative interaction terms (sex $x$ BPA) was also tested.

We performed a few sensitivity analyses. First, since fasting status ( $\geq 10$ or $<10$ hours) and time of urine collection (morning, afternoon, or evening) may be associated with urinary BPA levels, ${ }^{26,27}$ we assessed potential confounding by these variables. Second, we restricted our models to respondents aged 18 to 64 years, as standard BMI and waist circumference classifications may not be applicable to elderly adults. $^{24}$ Third, we re-ran the models excluding respondents with self-reported health professional-diagnosed chronic conditions (cardiovascular disease [including heart attack, stroke, and any heart disease], diabetes, and/or kidney disease) that may be related to obesity and BPA exposure/excretion. ${ }^{28,29}$ Fourth, since the distribution of full-sample urinary BPA quartiles differed between males and females, we repeated sex-stratified models using sex-specific instead of full-sample quartiles.

\section{Results}

Characteristics of the study population, including urinary BPA concentrations, are presented in Table 1. Overall, the GM urinary BPA concentration was $1.17 \mu \mathrm{g} / \mathrm{L}$

TABLE 2

Associations between urinary bisphenol $A$ and overweight and obesity (vs. under/normal weight) in adults 18 to 79 years of age, overall and by sex, 2007-2011 Canadian Health Measures Survey

\begin{tabular}{|c|c|c|c|c|c|c|c|c|c|c|}
\hline \multirow{3}{*}{ Urinary BPA concentration } & \multicolumn{5}{|c|}{ Model $1^{\mathrm{a}}$} & \multicolumn{5}{|c|}{ Model $2^{b}$} \\
\hline & \multirow{2}{*}{$\mathbf{N}$} & \multicolumn{2}{|c|}{ Overweight } & \multicolumn{2}{|c|}{ Obesity } & \multirow{2}{*}{$\mathbf{N}$} & \multicolumn{2}{|c|}{ Overweight } & \multicolumn{2}{|c|}{ Obesity } \\
\hline & & OR & $95 \% \mathrm{Cl}$ & OR & $95 \% \mathrm{Cl}$ & & OR & $95 \% \mathrm{Cl}$ & OR & $95 \% \mathrm{Cl}$ \\
\hline \multicolumn{11}{|l|}{ Overall } \\
\hline Quartile $1(<0.7 \mu \mathrm{g} / \mathrm{L})$ & 1345 & 1.00 & Ref & 1.00 & Ref & 1342 & 1.00 & Ref & 1.00 & Ref \\
\hline Quartile $2(0.7-1.2 \mu \mathrm{g} / \mathrm{L})$ & 1010 & 0.94 & $0.59-1.50$ & 1.05 & $0.71-1.54$ & 1009 & 0.91 & $0.58-1.44$ & 1.01 & $0.69-1.48$ \\
\hline Quartile 4 (> $2.4 \mu \mathrm{g} / \mathrm{L})$ & 1157 & 1.18 & $0.76-1.83$ & 1.56 & $1.02-2.38$ & 1156 & 1.14 & $0.73-1.77$ & 1.54 & $1.002-2.37$ \\
\hline$p$-trend ${ }^{c}$ & & \multicolumn{2}{|c|}{.322} & \multicolumn{2}{|c|}{.036} & & \multicolumn{2}{|c|}{.394} & \multicolumn{2}{|r|}{.041} \\
\hline Log BPA (continuous) & 4722 & 1.05 & $0.90-1.22$ & 1.15 & $1.01-1.31$ & 4715 & 1.03 & $0.89-1.20$ & 1.15 & $1.004-1.31$ \\
\hline \multicolumn{11}{|l|}{ Males } \\
\hline Quartile 4 (> $2.4 \mu \mathrm{g} / \mathrm{L})$ & 618 & 1.51 & $0.81-2.82$ & 1.77 & $0.78-4.01$ & 618 & 1.43 & $0.74-2.74$ & 1.60 & $0.67-3.80$ \\
\hline$p$-trend ${ }^{c}$ & & \multicolumn{2}{|c|}{.208} & \multicolumn{2}{|c|}{.193} & & \multicolumn{2}{|c|}{.248} & \multicolumn{2}{|r|}{.266} \\
\hline Log BPA (continuous) & 2273 & 1.17 & $0.95-1.45$ & 1.19 & $0.94-1.52$ & 2271 & 1.16 & $0.93-1.44$ & 1.17 & $0.91-1.51$ \\
\hline \multicolumn{11}{|l|}{ Females } \\
\hline Quartile $1(<0.7 \mu \mathrm{g} / \mathrm{L})$ & 808 & 1.00 & Ref & 1.00 & Ref & 805 & 1.00 & Ref & 1.00 & Ref \\
\hline Quartile $2(0.7-1.2 \mu \mathrm{g} / \mathrm{L})$ & 527 & 0.83 & $0.42-1.65$ & 0.90 & $0.49-1.66$ & 526 & 0.84 & $0.42-1.66$ & 0.93 & $0.52-1.68$ \\
\hline Quartile $3(1.3-2.4 \mu \mathrm{g} / \mathrm{L})$ & 575 & 0.86 & $0.45-1.64$ & 1.12 & $0.56-2.23$ & 575 & 0.86 & $0.44-1.67$ & 1.16 & $0.60-2.27$ \\
\hline Quartile 4 (> $2.4 \mu \mathrm{g} / \mathrm{L})$ & 539 & 0.95 & $0.49-1.85$ & 1.41 & $0.72-2.75$ & 538 & 0.93 & $0.46-1.86$ & 1.47 & $0.77-2.81$ \\
\hline
\end{tabular}

Abbreviations: $\mathrm{BPA}$, bisphenol A; $\mathrm{Cl}$, confidence interval; OR, odds ratio; Ref, reference category.

a Adjusted for sex (overall model only), age, and urinary creatinine concentration.

${ }^{\mathrm{b}}$ Adjusted for Model 1 covariates plus race/ethnicity, alcohol consumption, and junk food consumption.

${ }^{c} p$-value for test of trend calculated by modelling the median of each BPA quartile as a continuous variable. 
(95\% confidence interval [CI]: 1.10-1.24). GMs decreased with age and were significantly higher among males and those who consumed junk food $\geq 5$ times/week (all $p<.05$; data not shown). With the exception of sex $(p=.023)$, the distribution of BPA quartiles did not differ by any of the characteristics.

As shown in Table 1, $60 \%$ of Canadian adults were overweight $(35 \%)$ or obese $(25 \%)$, and $35 \%$ had an elevated waist circumference (i.e. centrally obese). GM urinary BPA concentrations increased with BMI, from $0.84 \mu \mathrm{g} / \mathrm{L}$ (95\% CI: 0.421.67 ) and $1.09 \mu \mathrm{g} / \mathrm{L}$ (95\% CI: 0.96-1.23) among underweight and normal weight individuals, respectively, to $1.34 \mu \mathrm{g} / \mathrm{L}$ (95\% CI: 1.13-1.59) among those in the class II/III obese categories ( $p$-trend $=0.06$ ). BPA concentrations did not differ by waist circumference category.

Table 2 presents associations of urinary BPA with BMI-defined overweight and obesity, overall and by sex. In the overall model adjusted for sex, age, and urinary creatinine (model 1), respondents in the highest BPA quartile had a significantly higher odds of being obese (vs. under/ normal weight) compared to those in the lowest quartile $(\mathrm{OR}=1.56,95 \% \mathrm{CI}$ : $1.02-$ 2.38), with an increasing trend across increasing quartiles ( $p$-trend $=.036$ ). Results remained largely unchanged following additional adjustment for potential confounders (model 2) (OR [quartile 4 vs. 1] $=1.54,95 \% \mathrm{CI}: 1.002-2.37$; $p$-trend $=$ .041). Similarly, natural log-transformed BPA (continuous) was positively associated with obesity $(\mathrm{OR}=1.15,95 \% \mathrm{CI}$ : 1.004-1.31). For the overweight category, associations were generally positive but nonsignificant, with an OR of 1.14 (95\% CI: 0.73-1.77) in the highest (vs. lowest) BPA quartile. When results were examined by sex, associations between urinary BPA and both overweight and obesity did not reach statistical significance in either sex. Although there was no evidence of a significant sex $\times$ BPA interaction $(p>.05$; data not shown), we observed stronger positive associations in males than females for both overweight (OR [quartile 4 vs. 1] $=1.43$ vs. 0.93 ) and obesity (OR [quartile 4 vs. 1] $=1.60$ vs. 1.47 ).

We did not find significant associations between urinary BPA and central obesity, overall (OR [quartile 4 vs. 1] $=1.16,95 \%$ CI: $0.81-1.66$; $p$-trend $=.463$ ) or by sex
(Table 3). Sex-stratified models suggested a stronger association in males than females (OR [quartile 4 vs. 1] $=1.28$ vs. 1.03), although a statistically significant interaction was not found $(p>.05$; data not shown). Additional adjustment for height in the models to control for overall stature did not alter the results (data not shown).

Figure 1 presents associations of urinary BPA quartile with continuous measures of BMI and waist circumference. Respondents in the third and fourth BPA quartiles had significantly greater BMI $\left(1.03 \mathrm{~kg} / \mathrm{m}^{2}\right.$, $95 \%$ CI: $0.30-1.76$; and $1.06 \mathrm{~kg} / \mathrm{m}^{2}, 95 \%$ CI: $0.18-1.93$, respectively) compared to those in the first quartile. A similar pattern was observed for waist circumference, although statistical significance was reached in the third $(2.42 \mathrm{~cm}, 95 \% \mathrm{CI}$ : 0.46-4.39) but not the fourth $(2.73 \mathrm{~cm}$, $95 \%$ CI: -0.14 to 5.60 ) quartile. Furthermore, each natural-log increase in urinary BPA concentration was associated with a $0.33 \mathrm{~kg} / \mathrm{m}^{2}$ (95\% CI: 0.10-0.57) increase in BMI and a $1.00 \mathrm{~cm}$ (95\% CI: 0.34-1.65) increase in waist circumference (Table 4), with slightly stronger associations in females ( $p$ for interaction $>.05$; data not shown).

In our sensitivity analyses (data available by request), inclusion of fasting status or time of urine collection did not change effect estimates by $>10 \%$ in any of the models, indicating that these variables did not confound the associations observed. Next, analyses restricted to adults aged 18 to 64 years yielded similar results, with

TABLE 3

Associations between urinary bisphenol $A$ and central obesity in adults 18 to 79 years of age, overall and by sex, 2007-2011 Canadian Health Measures Survey

\begin{tabular}{|c|c|c|c|c|c|c|}
\hline \multirow{2}{*}{ Urinary BPA concentration } & \multicolumn{3}{|c|}{ Model $1^{b}$} & \multicolumn{3}{|c|}{ Model $2^{c}$} \\
\hline & $\mathbf{N}$ & OR & $95 \% \mathrm{Cl}$ & $\mathbf{N}$ & OR & $95 \% \mathrm{Cl}$ \\
\hline \multicolumn{7}{|l|}{ Overall } \\
\hline Quartile $1(<0.7 \mu \mathrm{g} / \mathrm{L})$ & 1342 & 1.00 & Ref & 1339 & 1.00 & Ref \\
\hline Quartile $2(0.7-1.2 \mu \mathrm{g} / \mathrm{L})$ & 1003 & 1.12 & $0.76-1.67$ & 1001 & 1.12 & $0.75-1.65$ \\
\hline Quartile $3(1.3-2.4 \mu \mathrm{g} / \mathrm{L})$ & 1211 & 0.96 & $0.64-1.45$ & 1209 & 0.99 & $0.67-1.46$ \\
\hline Quartile 4 (> $2.4 \mu \mathrm{g} / \mathrm{L})$ & 1156 & 1.16 & $0.81-1.65$ & 1155 & 1.16 & $0.81-1.66$ \\
\hline$p$-trend ${ }^{d}$ & \multicolumn{3}{|c|}{.470} & & \multicolumn{2}{|c|}{.463} \\
\hline Log BPA (continuous) & 4712 & 1.06 & $0.96-1.16$ & 4704 & 1.05 & $0.96-1.16$ \\
\hline \multicolumn{7}{|l|}{ Males } \\
\hline Quartile $1(<0.7 \mu \mathrm{g} / \mathrm{L})$ & 537 & 1.00 & Ref & 537 & 1.00 & Ref \\
\hline Quartile $2(0.7-1.2 \mu \mathrm{g} / \mathrm{L})$ & 479 & 1.41 & $0.88-2.26$ & 478 & 1.27 & $0.80-2.02$ \\
\hline Quartile $3(1.3-2.4 \mu \mathrm{g} / \mathrm{L})$ & 636 & 1.31 & $0.86-1.99$ & 634 & 1.32 & 0.89-1.98 \\
\hline Quartile 4 (> $2.4 \mu \mathrm{g} / \mathrm{L})$ & 618 & 1.41 & $0.78-2.55$ & 618 & 1.28 & $0.72-2.27$ \\
\hline$p$-trend ${ }^{d}$ & \multicolumn{3}{|c|}{.434} & & \multicolumn{2}{|c|}{.616} \\
\hline Log BPA (continuous) & 2270 & 1.08 & $0.92-1.28$ & 2267 & 1.07 & $0.91-1.26$ \\
\hline \multicolumn{7}{|l|}{ Females } \\
\hline Quartile $1(<0.7 \mu \mathrm{g} / \mathrm{L})$ & 805 & 1.00 & Ref & 802 & 1.00 & Ref \\
\hline Quartile $2(0.7-1.2 \mu \mathrm{g} / \mathrm{L})$ & 524 & 0.95 & $0.55-1.65$ & 523 & 1.01 & $0.57-1.77$ \\
\hline Quartile $3(1.3-2.4 \mu \mathrm{g} / \mathrm{L})$ & 575 & 0.76 & $0.43-1.36$ & 575 & 0.80 & $0.45-1.44$ \\
\hline Quartile 4 (> $2.4 \mu \mathrm{g} / \mathrm{L})$ & 538 & 1.00 & $0.60-1.66$ & 537 & 1.03 & $0.64-1.65$ \\
\hline$p$-trend ${ }^{d}$ & \multicolumn{3}{|c|}{.902} & & \multicolumn{2}{|c|}{.864} \\
\hline Log BPA (continuous) & 2442 & 1.03 & $0.87-1.18$ & 2437 & 1.03 & $0.90-1.18$ \\
\hline
\end{tabular}

Abbreviations: BPA, bisphenol A; CI, confidence interval; OR, odds ratio; Ref, reference category.

${ }^{a}$ Waist circumference $\geq 102 \mathrm{~cm}$ in males and $\geq 88 \mathrm{~cm}$ in females.

${ }^{\mathrm{b}}$ Adjusted for sex (overall model only), age, and urinary creatinine concentration.

' Adjusted for Model 1 covariates plus race/ethnicity, alcohol consumption, junk food consumption, and sugar-sweetened beverage consumption.

${ }^{d} p$-value for test of trend calculated by modelling the median of each BPA quartile as a continuous variable. 
FIGURE 1

Mean difference in (A) body mass index and (B) waist circumference across urinary bisphenol A quartiles (vs. quartile 1) in adults 18 to 79 years of age, overall and by sex, 2007-2011 Canadian Health Measures Survey

A - Body mass index

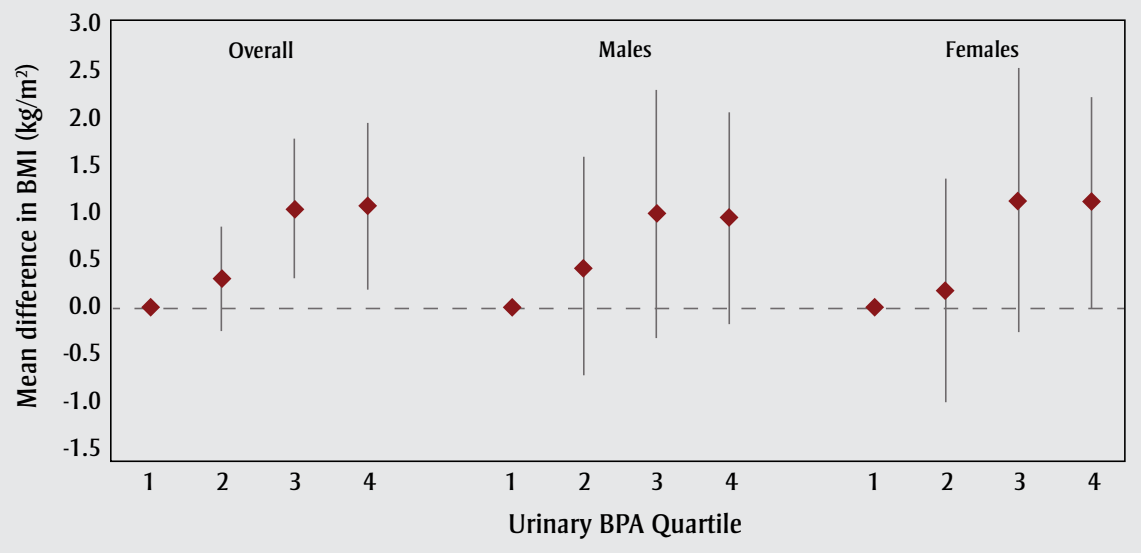

B - Waist circumference

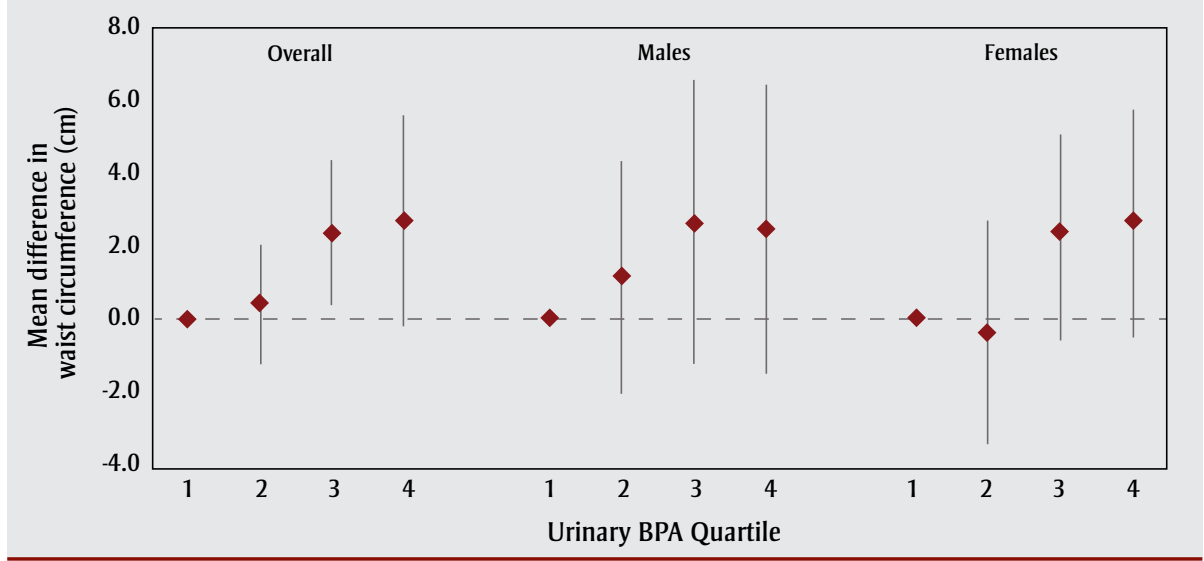

Abbreviations: BMI, body mass index; BPA, bisphenol A.

Notes: Quartile 1: $<0.7 \mu \mathrm{g} / \mathrm{L}$; quartile 2: 0.7-1.2 $\mu \mathrm{g} / \mathrm{L}$; quartile 3: 1.3-2.4 $\mu \mathrm{g} / \mathrm{L}$; quartile 4: > $2.4 \mu \mathrm{g} / \mathrm{L}$.

All models adjusted for sex (overall models only), age, urinary creatinine concentration, race/ethnicity, alcohol consumption, and junk food consumption. Models for waist circumference additionally adjusted for sugar-sweetened beverage consumption. Error bars represent $95 \%$ confidence intervals.

slightly stronger associations for obesity (OR [quartile 4 vs. 1] = 1.64, 95\% CI: 1.01-2.66; $p$-trend $=.018$ ), as compared to analyses conducted on the full sample. Similarly, when we restricted our analyses to respondents without specific chronic conditions, results did not change appreciably. Finally, when sex-stratified analyses were repeated using sex-specific instead of full-sample quartiles, effect estimates changed slightly (e.g. OR for obesity was attenuated in both sexes for quartile 4 vs .1) but were generally in the same direction, with stronger positive associations consistently observed in males.

\section{Discussion}

Using data from the CHMS, our study provides, for the first time, an examination of associations between BPA and indicators of obesity in the general Canadian adult population. We found that urinary BPA was positively associated with BMIdefined general obesity in a dose-dependent manner and that this association persisted after controlling for potential confounders, including diet-related factors. These findings are consistent with other large-scale cross-sectional studies of adults in the USA ${ }^{11,12}$ and China, ${ }^{13}$ as well as with studies in children. ${ }^{30-33}$ Effect sizes in our study were slightly lower than those reported using the US National Health and Nutrition Examination Survey ${ }^{11}$ (OR [quartile 4 vs. 1] = 1.76) and were more similar with the study in Chinese adults $^{13}$ (OR [quartile 4 vs. 1] = 1.50). A possible explanation for the weaker associations, compared to US results, may be related to the substantially lower BPA concentrations in our study and the Chinese study. In addition, while our results demonstrated increasing odds of obesity across increasing urinary BPA quartiles, some studies reported a potential threshold or non-monotonic effect. ${ }^{11,15,32}$ Although non-monotonic associations have been commonly observed for BPA and other endocrine disruptors in toxicological studies, $^{34}$ ubiquitous low-level exposures to BPA in human populations present a challenge for assessing and interpreting doseresponse relationships. ${ }^{35}$

Consistent with previous studies, ${ }^{11,13,14}$ our study also provides evidence for positive associations between urinary BPA and continuous BMI and waist circumference measures. However, unlike studies in the USA $^{11,12}$ and Asia, ${ }^{13,14}$ we did not find an association between urinary BPA and central obesity defined using waist circumference cut-offs. Although we used the same cut-offs as the US studies, differences in racial/ethnic makeup between the populations may have contributed to the discrepant findings. ${ }^{36}$ Furthermore, it has been shown that urinary BPA concentration differed by race/ethnicity and was significantly higher among black compared to white Americans. ${ }^{27}$ However, owing to small sample sizes of individual ethnic groups within our study population, we could not further control for race/ethnicity or examine race/ethnicity-specific associations.

Several biological mechanisms have been proposed to explain the association between BPA and obesity, although the exact mode of action remains unclear. For example, BPA has been shown to promote adipocyte differentiation and fat accumulation, ${ }^{9}$ as well as bind to estrogen receptors on adipocytes and inhibit the release of the hormone adiponectin. ${ }^{37}$ While most animal studies focused on in utero exposure, Miyawaki et al. ${ }^{10}$ showed that BPA exposure during both perinatal and postnatal periods led to weight gain in mice. Additionally, long-term exposure to BPA in adult mice was shown to increase adipose tissue mass and induce insulin resistance, hyperglycemia, and hypercholesterolemia, ${ }^{38}$ suggesting the role of BPA in the development of obesity and cardiometabolic dysfunction. Similarly, epidemiological studies provide growing evidence that, in addition to obesity, exposure to BPA may be associated with increased risk of diabetes and cardiovascular disorders. ${ }^{39}$ 
TABLE 4

Associations between log-transformed urinary bisphenol $A$ and continuous measures of body mass index and waist circumference in adults 18 to 79 years of age, overall and by sex, 2007-2011 Canadian Health Measures Survey

\begin{tabular}{|c|c|c|c|c|c|c|}
\hline & \multicolumn{3}{|c|}{ Body mass index $\left(\mathbf{k g} / \mathrm{m}^{2}\right)^{a}$} & \multicolumn{3}{|c|}{ Waist circumference (cm) ${ }^{b}$} \\
\hline & $\mathbf{N}$ & $\beta^{c}$ & $95 \% \mathrm{Cl}$ & $\mathbf{N}$ & $\beta^{c}$ & $95 \% \mathrm{Cl}$ \\
\hline \multicolumn{7}{|l|}{ Overall } \\
\hline Log BPA (continuous) & 4715 & 0.33 & 0.10 to 0.57 & 4704 & 1.00 & 0.34 to 1.65 \\
\hline \multicolumn{7}{|l|}{ Males } \\
\hline Log BPA (continuous) & 2271 & 0.28 & -0.04 to 0.59 & 2267 & 0.72 & -0.22 to 1.67 \\
\hline \multicolumn{7}{|l|}{ Females } \\
\hline Log BPA (continuous) & 2444 & 0.38 & 0.73 to 2.28 & 2437 & 1.16 & 0.39 to 1.92 \\
\hline
\end{tabular}

Abbreviations: BPA, bisphenol A; $\mathrm{Cl}$, confidence interval.

a Adjusted for sex (overall model only), age, urinary creatinine concentration, race/ethnicity, alcohol consumption, and junk food consumption.

${ }^{\mathrm{b}}$ Adjusted for sex (overall model only), age, urinary creatinine concentration, race/ethnicity, alcohol consumption, junk food consumption, and sugar-sweetened beverage consumption.

' Mean change in body mass index $\left(\mathrm{kg} / \mathrm{m}^{2}\right)$ or waist circumference $(\mathrm{cm})$ per natural-log unit increase in urinary BPA concentration.

Like most epidemiological studies, we did not find evidence of effect modification by sex in the association between urinary BPA and obesity. Sex differences in the BPA-obesity association have been implicated in animal studies, ${ }^{16,40}$ possibly relating to differences in BPA metabolism ${ }^{41}$ and estrogen receptor expression, ${ }^{42}$ as well as sex-specific effects of BPA on dietary intake and energy expenditure. ${ }^{43}$ Given the biological plausibility, more research is needed to delineate potential sex differences in BPA-induced health outcomes.

\section{Strengths and limitations}

This is the first Canadian study to examine associations between BPA and indicators of obesity in adults. Strengths of our study include the population-based design, large sample size, direct anthropometric measurements, high-quality urinary BPA assays, and assessment of multiple potential confounders, including several dietary factors. This is important as recent systematic reviews have pointed out the lack of adjustment for diet, especially processed food consumption, as a major limitation of studies investigating associations between BPA and health outcomes. ${ }^{26,35,39}$

This study has several limitations. First, due to the cross-sectional nature of CHMS data, temporal relationships could not be established. It is possible that obese individuals store, metabolize, and/or excrete BPA differently from non-obese individuals, leading to higher BPA levels in their urine. ${ }^{44,45}$ Nonetheless, a recent prospective cohort study of women showed that higher urinary BPA concentration at baseline was associated with greater weight gain during a 10 -year follow-up, ${ }^{46}$ although additional longitudinal studies in both sexes are warranted. Second, single spot urine measures may not be representative of long-term exposure to BPA. Considerable within-person variability has been shown in urinary BPA measured throughout the day and week ${ }^{47}$ and over a period of 1 to 3 years, ${ }^{48}$ likely relating to variations in dietary intake. However, our sensitivity analyses showed that time of urine collection and fasting status did not confound the associations observed. Moreover, a recent panel study collected repeated measures of urinary BPA and BMI over a 3-year period and found significant positive associations with overweight in elderly adults regardless of whether single or average BPA measures were analyzed. ${ }^{15}$

\section{Conclusion}

In a nationally representative sample of Canadian adults, we showed that urinary BPA was positively associated with general obesity, as well as with continuous BMI and waist circumference. While the imbalance between energy intake and energy expenditure remains a major contributor to obesity, health and economic consequences of obesity attributable to BPA exposure should not be ignored. ${ }^{49}$ Furthermore, although BPA is not currently prohibited in Canada, except in baby bottles and cosmetic products, it has been recommended to limit BPA exposure from food packaging given potential effects on health outcomes such as obesity. ${ }^{6}$ This underscores the need to further explore the role of BPA as a potential environmental obesogen. Future studies should aim to collect prospective data with repeated measures over extended time periods in order to improve exposure classification and address the temporal relationship between BPA and obesity.

\section{Acknowledgements}

The CHMS was conducted by Statistics Canada in partnership with Health Canada and PHAC with funding from the Canadian federal government. No external funding was obtained for this study.

\section{Conflicts of interest}

The authors declare no conflicts of interest.

\section{Authors' contributions and statement}

MTD and VCC conceptualized the study, conducted the data analyses, interpreted the data, and drafted the manuscript. MAM and MdG contributed to the interpretation of data, provided expertise and guidance in specific areas, and critically reviewed and revised the manuscript.

The content and views expressed in this article are those of the authors and do not necessarily reflect those of the Government of Canada.

\section{References}

1. Jia H, Lubetkin EI. Trends in quality-adjusted life-years lost contributed by smoking and obesity. Am J Prev Med. 2010;38(2):138-44.

2. Manuel DG, Tuna M, Hennessy D, et al. Projections of preventable risks for cardiovascular disease in Canada to 2021: a microsimulation modelling approach. CMAJ Open. 2014;2(2): E94-101.

3. Bancej C, Jayabalasingham B, Wall RW, et al. Evidence brief - Trends and projections of obesity among Canadians. Health Promot Chronic Dis Prev Can. 2015;35(7):109-12. 
4. World Health Organization. Obesity: preventing and managing the global epidemic: report of a WHO consultation. Geneva, Switzerland: World Health Organization; 2000.

5. Baillie-Hamilton PF. Chemical toxins: a hypothesis to explain the global obesity epidemic. J Altern Complement Med. 2002;8(2):185-92.

6. Health Canada. Second report on human biomonitoring of environmental chemicals in Canada: results of the Canadian Health Measures Survey, cycle 2 (2009-2011). Ottawa (ON): Health Canada; 2013.

7. Bushnik T, Haines D, Levallois P, Levesque J, Van Oostdam J, Viau C. Lead and bisphenol A concentrations in the Canadian population. Health Rep. 2010;21(3):7-18.

8. Calafat AM, Ye X, Wong LY, Reidy JA, Needham LL. Exposure of the U.S. population to bisphenol A and 4-tertiary-octylphenol: 2003-2004. Environ Health Perspect. 2008;116(1):39-44.

9. Masuno H, Iwanami J, Kidani $\mathrm{T}$, Sakayama K, Honda K. Bisphenol A accelerates terminal differentiation of 3T3-L1 cells into adipocytes through the phosphatidylinositol 3-kinase pathway. Toxicol Sci. 2005;84(2): 319-27.

10. Miyawaki J, Sakayama K, Kato H, Yamamoto $\mathrm{H}$, Masuno $\mathrm{H}$. Perinatal and postnatal exposure to bisphenol A increases adipose tissue mass and serum cholesterol level in mice. J Atheroscler Thromb. 2007;14(5):245-52.

11. Carwile JL, Michels KB. Urinary bisphenol A and obesity: NHANES 2003-2006. Environ Res. 2011;111(6): 825-30.

12. Shankar A, Teppala S, Sabanayagam C. Urinary bisphenol A levels and measures of obesity: results from the National Health and Nutrition Examination Survey 2003-2008. ISRN Endocrinol. 2012;2012:965243.

13. Wang T, Li M, Chen B, et al. Urinary bisphenol A (BPA) concentration associates with obesity and insulin resistance. J Clin Endocrinol Metab. 2012; 97(2):E223-7.
14. Ko A, Hwang MS, Park JH, Kang HS, Lee HS, Hong JH. Association between urinary bisphenol A and waist circumference in Korean adults. Toxicol Res. 2014;30(1):39-44.

15. Lee MR, Kim JH, Choi YH, Bae S, Park C, Hong YC. Association of bisphenol A exposure with overweight in the elderly: a panel study. Environ Sci Pollut Res Int. 2015;22(12):9370-7.

16. Caporossi L, Papaleo B. Exposure to bisphenol A and gender differences: from rodents to human evidences and hypothesis about the health effects. J Xenobiotics. 2015;5(1):5264.

17. Statistics Canada. Canadian Health Measures Survey (CHMS) data user guide: cycle 1. Ottawa (ON): Statistics Canada; 2011.

18. Statistics Canada. Canadian Health Measures Survey (CHMS) data user guide: cycle 2. Ottawa (ON): Statistics Canada; 2013.

19. Statistics Canada. Instructions for combining cycle 1 and cycle 2 Canadian Health Measures Survey (CHMS) data. Ottawa (ON): Statistics Canada; 2014.

20. Institut national de santé publique du Québec. Analytical method for the determination of bisphenol A, triclosan, triclocarban and pesticide metabolites in urine by GC-MS-MS (E-454), condensed version for CHMS. Québec (QC): Laboratoire de toxicology; 2009.

21. Institut national de santé publique du Québec. Analytical method for the determination of urine creatinine on Hitachi 917 (C-530), condensed version. Québec (QC): Laboratoire de toxicologie; 2008.

22. Barr DB, Wilder LC, Caudill SP, Gonzalez AJ, Needham LL, Pirkle JL. Urinary creatinine concentrations in the U.S. population: implications for urinary biologic monitoring measurements. Environ Health Perspect. 2005; 113(2):192-200.

23. Canadian Society for Exercise Physiology. The Canadian Physical Activity, Fitness and Lifestyle Approach (CPAFLA), 3rd ed. Ottawa (ON): Canadian Society for Exercise Physiology; 2003.
24. Douketis JD, Paradis G, Keller H, Martineau C. Canadian guidelines for body weight classification in adults: application in clinical practice to screen for overweight and obesity and to assess disease risk. CMAJ. 2005;172(8):995-8.

25. Mendez MA, Popkin BM, Jakszyn P, et al. Adherence to a Mediterranean diet is associated with reduced 3-year incidence of obesity. J Nutr. 2006; 136(11):2934-8.

26. LaKind JS, Goodman M, Mattison DR. Bisphenol A and indicators of obesity, glucose metabolism/type 2 diabetes and cardiovascular disease: a systematic review of epidemiologic research. Crit Rev Toxicol. 2014; 44(2):121-50.

27. LaKind JS, Levesque J, Dumas $P$, Bryan S, Clarke J, Naiman DQ. Comparing United States and Canadian population exposures from National Biomonitoring Surveys: bisphenol A intake as a case study. J Expo Sci Environ Epidemiol. 2012; 22(3):219-26.

28. Lang IA, Galloway TS, Scarlett A, et al. Association of urinary bisphenol A concentration with medical disorders and laboratory abnormalities in adults. JAMA. 2008;300(11):1303-10.

29. You L, Zhu X, Shrubsole MJ, et al. Renal function, bisphenol A, and alkylphenols: results from the National Health and Nutrition Examination Survey (NHANES 2003-2006). Environ Health Perspect. 2011;119(4):527-33.

30. Bhandari R, Xiao J, Shankar A. Urinary bisphenol A and obesity in U.S. children. Am J Epidemiol. 2013; 177(11):1263-70.

31. Eng DS, Lee JM, Gebremariam A, Meeker JD, Peterson K, Padmanabhan $\mathrm{V}$. Bisphenol A and chronic disease risk factors in U.S. children. Pediatrics. 2013;132(3):E637-45.

32. Trasande L, Attina TM, Blustein J. Association between urinary bisphenol A concentration and obesity prevalence in children and adolescents. JAMA. 2012;308(11):1113-21.

33. Li DK, Miao M, Zhou Z, et al. Urine bisphenol-A level in relation to obesity and overweight in school-age children. PLoS One. 2013;8(6):E65399. 
34. Vandenberg LN. Non-monotonic dose responses in studies of endocrine disrupting chemicals: bisphenol $\mathrm{A}$ as a case study. Dose Response. 2013; 12(2):259-76.

35. Oppeneer SJ, Robien K. Bisphenol A exposure and associations with obesity among adults: a critical review. Public Health Nutr. 2015;18(10): 1847-63.

36. Lear SA, James PT, Ko GT, Kumanyika S. Appropriateness of waist circumference and waist-to-hip ratio cutoffs for different ethnic groups. Eur J Clin Nutr. 2010;64(1):42-61.

37. Hugo ER, Brandebourg TD, Woo JG, Loftus J, Alexander JW, Ben-Jonathan N. Bisphenol A at environmentally relevant doses inhibits adiponectin release from human adipose tissue explants and adipocytes. Environ Health Perspect. 2008;116(12):1642-7.

38. Marmugi A, Lasserre F, Beuzelin D, et al. Adverse effects of long-term exposure to bisphenol A during adulthood leading to hyperglycaemia and hypercholesterolemia in mice. Toxicology. 2014;325:133-43.

39. Rancière F, Lyons JG, Loh VH, et al. Bisphenol A and the risk of cardiometabolic disorders: a systematic review with meta-analysis of the epidemiological evidence. Environ Health. 2015;14:46.

40. vom Saal FS, Nagel SC, Coe BL, Angle $\mathrm{BM}$, Taylor JA. The estrogenic endocrine disrupting chemical bisphenol A (BPA) and obesity. Mol Cell Endocrinol. 2012;354(1-2):74-84.

41. Takeuchi T, Tsutsumi O, Nakamura $\mathrm{N}$, et al. Gender difference in serum bisphenol A levels may be caused by liver UDP-glucuronosyltransferase activity in rats. Biochem Biophys Res Commun. 2004;325(2):549-54.

42. Davis KE, Neinast MD, Sun K, et al. The sexually dimorphic role of adipose and adipocyte estrogen receptors in modulating adipose tissue expansion, inflammation, and fibrosis. Mol Metab. 2013;2(3):227-42.
43. Mackay H, Patterson ZR, Khazall R, Patel S, Tsirlin D, Abizaid A. Organizational effects of perinatal exposure to bisphenol-A and diethylstilbestrol on arcuate nucleus circuitry controlling food intake and energy expenditure in male and female CD-1 mice. Endocrinology. 2013;154(4): 1465-75.

44. Hays SM, Aylward LL, Blount BC. Variation in urinary flow rates according to demographic characteristics and body mass index in NHANES: potential confounding of associations between health outcomes and urinary biomarker concentrations. Environ Health Perspect. 2015;123(4):293-300.

45. Geens $T$, Neels $H$, Covaci A. Distribution of bisphenol-A, triclosan and n-nonylphenol in human adipose tissue, liver and brain. Chemosphere. 2013;87(7):796-802.

46. Song Y, Hauser R, Hu FB, Franke AA, Liu S, Sun Q. Urinary concentrations of bisphenol A and phthalate metabolites and weight change: a prospective investigation in US women. Int $\mathrm{J}$ Obes (Lond). 2014;38(12):1532-7.

47. Ye X, Wong LY, Bishop AM, Calafat AM. Variability of urinary concentrations of bisphenol A in spot samples, first morning voids, and 24-hour collections. Environ Health Perspect. 2011;119(7):983-8.

48. Townsend MK, Franke AA, Li X, Hu FB, Eliassen AH. Within-person reproducibility of urinary bisphenol A and phthalate metabolites over a 1 to 3 year period among women in the Nurses' Health Studies: a prospective cohort study. Environ Health. 2013; 12(1):80.

49. Trasande L. Further limiting bisphenol A in food uses could provide health and economic benefits. Health Aff. 2014;33(2):316-23. 